TITLE:

\title{
Spin-singlet Bose-Einstein condensation of two-electron atoms
}

\section{$\operatorname{AUTHOR}(S):$}

Takasu, Y; Maki, K; Komori, K; Takano, T; Honda, K; Kumakura, M; Yabuzaki, T; Takahashi, Y

\section{CITATION:}

Takasu, Y ...[et al]. Spin-singlet Bose-Einstein condensation of twoelectron atoms. PHYSICAL REVIEW LETTERS 2003, 91(4): 040404.

\section{ISSUE DATE:}

2003-07-25

URL:

http://hdl.handle.net/2433/49884

RIGHT:

Copyright 2003 American Physical Society 


\title{
Spin-Singlet Bose-Einstein Condensation of Two-Electron Atoms
}

\author{
Yosuke Takasu, Kenichi Maki, Kaduki Komori, Tetsushi Takano, Kazuhito Honda, ${ }^{*}$ Mitsutaka Kumakura, \\ Tsutomu Yabuzaki, and Yoshiro Takahashi ${ }^{\dagger}$ \\ Department of Physics, Graduate School of Science, Kyoto University, Japan 606-8502
}

(Received 30 April 2003; published 25 July 2003)

\begin{abstract}
We report the observation of a Bose-Einstein condensation of ytterbium atoms by evaporative cooling in a novel crossed optical trap. Unlike the previously observed condensates, a ytterbium condensate is a two-electron system in a singlet state and has distinct features such as the extremely narrow intercombination transitions which are ideal for future optical frequency standard and the insensitivity to external magnetic field which is important for precision coherent atom optics, and the existence of the novel metastable triplet states generated by optical excitation from the singlet state.
\end{abstract}

DOI: 10.1103/PhysRevLett.91.040404

PACS numbers: 03.75.Hh, 05.30.Jp, 32.80.Pj

Bose-Einstein condensation (BEC) in ultracold weakly interacting dilute gases is undoubtedly one of the most interesting research fields. Thus far only condensates of the atoms with electron spins have been produced $(\mathrm{Rb}$ [1,2], Na [3], Li [4], K [5], Cs [6], H [7], and metastable triplet $\mathrm{He}[8,9])$. Extending atomic species beyond these atoms is an important step for a future investigation. In particular, several groups are involved in the search for $\mathrm{BEC}$ of atoms with no electron spins in two-electron systems such as alkaline-earth and ytterbium $(\mathrm{Yb})$ atoms. $\mathrm{Yb}$ is a rare earth but its electronic structure in the ground state is $(\mathrm{Xe}) 4 f^{14} 6 s^{2}\left({ }^{1} \mathrm{~S}_{0}\right)$ similar to the alkaline-earth atoms.

In this Letter, we report the achievement of BEC of ${ }^{174} \mathrm{Yb}$ atoms. Unlike the previously observed condensates, $\mathrm{Yb}$ BEC has distinct features connected to the two valence electrons. A BEC of such two-electron systems [10] is remarkable because it offers many important possibilities for fundamental researches and applications. The two valence electrons result in singlet and triplet states connected by extremely narrow intercombination transitions which have received considerable attention as a frequency and time standard with unprecedented precision [11]. The simple structure of the spin-zero ground state ${ }^{1} \mathrm{~S}_{0}$ with no nuclear spins results in spinless BEC, which is considered a dilute gas analog of the well-known superfluid ${ }^{4} \mathrm{He}$. The spinless BEC is insensitive to external magnetic field, which is invaluable in precision coherent atom interferometry. The spinless BEC has also an advantage in the atom manipulation near the surface due to the suppressed heating in the vicinity of the surface [12]. Optical excitation of a BEC to a metastable ${ }^{3} \mathrm{P}_{2}$ state offers another interesting example of a metastable state BEC $[8,9]$. A BEC in the ${ }^{3} \mathrm{P}_{2}$ state for the alkaline-earthlike atoms is especially interesting because a new type of purely long-range molecular state arising due to an interplay of a quadrupole interaction and the magnetic field leads to a resonant behavior of the scattering length [13].

In addition to these distinct features connected to the two-electron systems, $\mathrm{Yb}$ has another great advantage due to the existence of rich varieties of stable isotopes of five bosons $\left({ }^{168} \mathrm{Yb},{ }^{170} \mathrm{Yb},{ }^{172} \mathrm{Yb},{ }^{174} \mathrm{Yb}\right.$, and $\left.{ }^{176} \mathrm{Yb}\right)$ and two fermions ( ${ }^{171} \mathrm{Yb}$ and ${ }^{173} \mathrm{Yb}$ ), which allows us to study various interesting quantum degenerate gases of $\mathrm{Yb}$ atoms. A straightforward application of the present scheme to other bosonic isotopes would result in the creation of $\mathrm{Yb}$ BEC with possibly various values of scattering lengths. The combination of the present method with sympathetic cooling technique $[5,14,15]$ would enable us to create mixtures of BEC and Fermi degeneracy in an optical trap as well. In fact, the sympathetic cooling effect between bosonic ${ }^{174} \mathrm{Yb}$ and fermionic ${ }^{171} \mathrm{Yb}$ has already been observed using the thermal gases trapped at a high density [16].

The feature of the spinless ground state, however, has prevented these atoms from the realization of BEC in a conventional recipe of BEC production using a magnetic trap and evaporative cooling by a radio frequency knife. Therefore an all-optical scheme [17] is the unique way to the realization of BEC. We have achieved the Yb BEC by all-optical means in a novel configuration of crossed faroff resonant trap (FORT). Through evaporative cooling of ${ }^{174} \mathrm{Yb}$ atoms by gradually ramping down the trap depth, we have observed anisotropic expansion after release from the trap, which is characteristic of BEC. From this behavior, we have estimated the scattering length of ${ }^{174} \mathrm{Yb}$.

The experimental apparatus is based on a magnetooptical trap (MOT) with the weakly allowed intercombination transition [18] and a crossed FORT system [19]. Yb atoms in a thermal beam are first slowed by Zeeman slower technique with the strong singlet transition $\left({ }^{1} \mathrm{~S}_{0}-\right.$ ${ }^{1} \mathrm{P}_{1}$, the wavelength is $399 \mathrm{~nm}$, the linewidth is $29 \mathrm{MHz}$, and the saturation intensity is $60 \mathrm{~mW} / \mathrm{cm}^{2}$ ), and then loaded into the MOT with the intercombination transition ${ }^{1} \mathrm{~S}_{0}-{ }^{3} \mathrm{P}_{1}$, the wavelength is $556 \mathrm{~nm}$, the linewidth is $182 \mathrm{kHz}$, and the saturation intensity is $0.14 \mathrm{~mW} / \mathrm{cm}^{2}$ ). This scheme allows us to collect a large number of atoms in microkelvin range. The FORT beam is generated from a cw green diode-pumped solid state laser whose 
wavelength is $532 \mathrm{~nm}$ and power is $10 \mathrm{~W}$. The generated beam is split by a polarizing beam splitter into two beams, which pass through respective acousto-optic modulators for independent control of the beam powers. The beams are frequency-shifted relative to each other with the acousto-optic modulators in order to avoid interference between the beams. The beams are tightly focused and crossed at their foci. One beam is in vertical direction and the other is inclined at $8^{\circ}$ from the horizontal direction. For detection, absorption images are taken with a horizontally propagating probe beam resonant with the singlet transition. The fact that only the visible laser sources are required in our experiment is also an advantage over previous all-optical formations of BEC [6,17].

The experimental sequence begins with the loading of Zeeman-slowed $\mathrm{Yb}$ atoms in the MOT using intercombination transition in $20 \mathrm{~s}$. About $10^{7}$ atoms are loaded into the MOT, and the temperature is about $40 \mu \mathrm{K}$. The FORT beams are turned on during this phase, and a fraction of atoms in the MOT are transferred into the FORT. About $10^{6}$ atoms are trapped in the FORT, and the temperature of the trapped atoms is $180 \mu \mathrm{K}$. The crossed FORT with almost equal trap depths results in an extremely high density of trapped ${ }^{174} \mathrm{Yb}$ atoms of more than $10^{14} \mathrm{~cm}^{-3}$ [19]. This configuration, however, does not lead to efficient evaporative cooling due to the rapid decrease of the number of trapped atoms. A successful evaporative cooling result is obtained for the configuration of the crossed

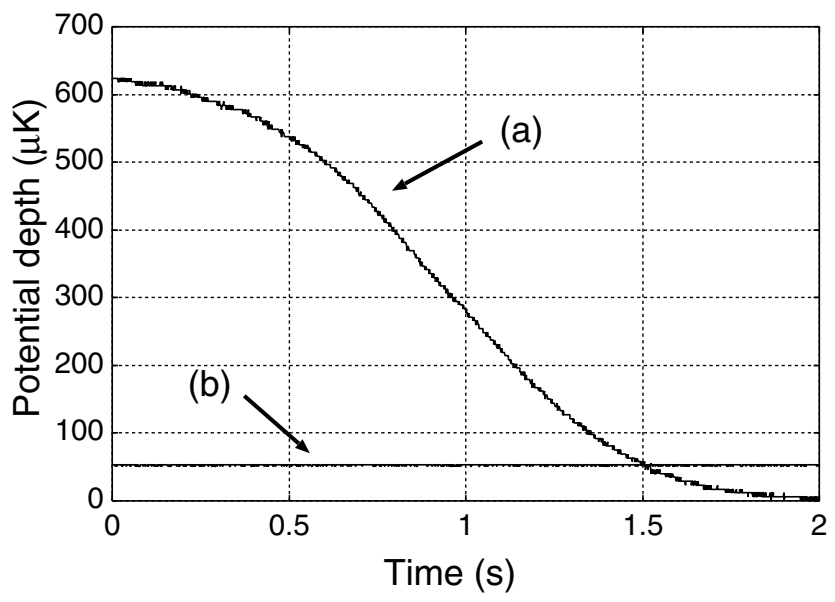

FIG. 1. Potential depths induced by (a) the horizontal FORT beam and (b) the vertical FORT beam, which are calculated from the beam waists and the peak intensities of trap beams, as a function of time in evaporative cooling. It is to be noted that the vertical gradient of the vertical FORT beam is too small to support atoms against the gravity field, and thus the trap depth along the vertical direction is mostly determined by the horizontal beam. The evaporative cooling is carried out by gradually decreasing the potential depth due to the horizontal FORT beam from 630 to $2.2 \mu \mathrm{K}$ in $2 \mathrm{~s}$ while keeping the potential due to the vertical FORT beam constant of $53 \mu \mathrm{K}$.
FORT with greatly asymmetrical trap depths. The horizontal and vertical FORT beam powers are 5.0 and $0.2 \mathrm{~W}$, respectively. The horizontal and vertical FORT beam waists are 14 and $11 \mu \mathrm{m}$, respectively. The potential depths induced by the horizontal beam and the vertical beam, which are calculated from the beam waists and the peak intensities, are 630 and $53 \mu \mathrm{K}$, respectively. It is to be noted that, because the vertical gradient of the vertical beam is too small to support atoms against the gravity field, the atoms are initially trapped mainly in the horizontal trap, and the trap depth along the vertical direction is mostly determined by the horizontal beam.

Evaporative cooling is carried out by gradually decreasing the horizontal FORT power in $2 \mathrm{~s}$ while keeping the vertical beam power constant (see Fig. 1). Although almost all atoms are initially trapped in the horizontal FORT, the atoms become concentrated into the crossed region as the horizontal FORT power decreases. This situation is quite similar to the delayed crossed FORT developed in our previous work [19]. When the final horizontal power is a little higher than $34 \mathrm{~mW}$, the isotropic expansion of released atoms is observed [see Fig. 2(b)]. In contrast, when the final horizontal power is decreased to $34 \mathrm{~mW}$, which corresponds to the $2.2 \mu \mathrm{K}$ potential depth, anisotropic expansion after release from

(a)
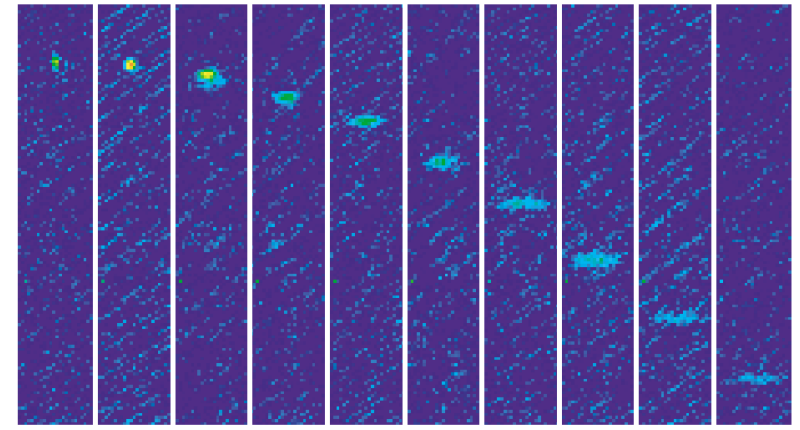

(b)
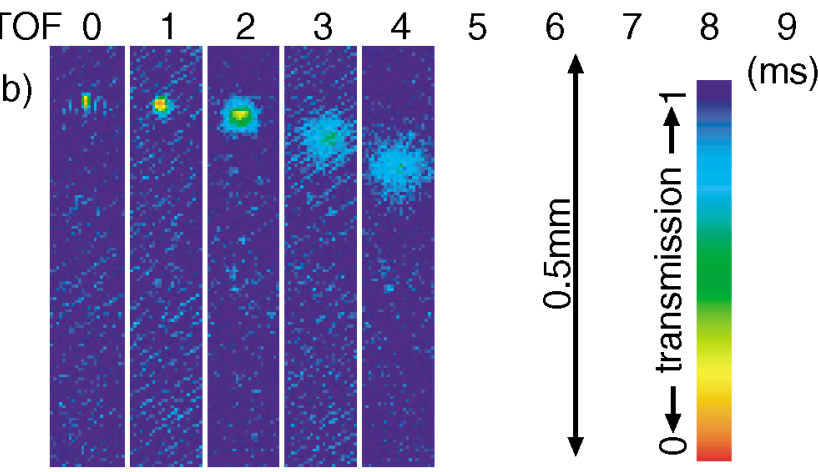

FIG. 2 (color). Pseudocolor absorption images of ${ }^{174} \mathrm{Yb}$ atoms as a function of time after release from the trap whose trap depths are (a) $2.2 \mu \mathrm{K}$ and (b) $6.7 \mu \mathrm{K}$, respectively. In (a) the vertical widths remain nearly constant while the horizontal widths increase rapidly because of anisotropic trap confinement. In contrast, atoms are expanded isotropically in (b). The temperature is $0.9 \mu \mathrm{K}$. 
the trap has appeared [see Fig. 2(a)]. In particular, the atom cloud changes from a prolate shape immediately after release from the trap, reflecting the stronger horizontal confinement, to oblate shapes at later flight times, which is a clear signature of BEC of asymmetrical trap. The trapped atom number $N$ is typically $5 \times 10^{3}$.

The atom density distributions along the vertical direction after $5 \mathrm{~ms}$ of free expansion are shown in Fig. 3 together with the absorption images. The Gaussian fits which are shown as red broken lines in Fig. 3(b) obviously show the bimodal nature of the momentum distributions just below the BEC transition temperature. Broad and narrow distributions correspond to the thermal and condensate gases, respectively. Figure 3(a) shows a pure condensate, and Fig. 3(c) shows a pure thermal cloud of $T=0.79 \mu \mathrm{K}$.

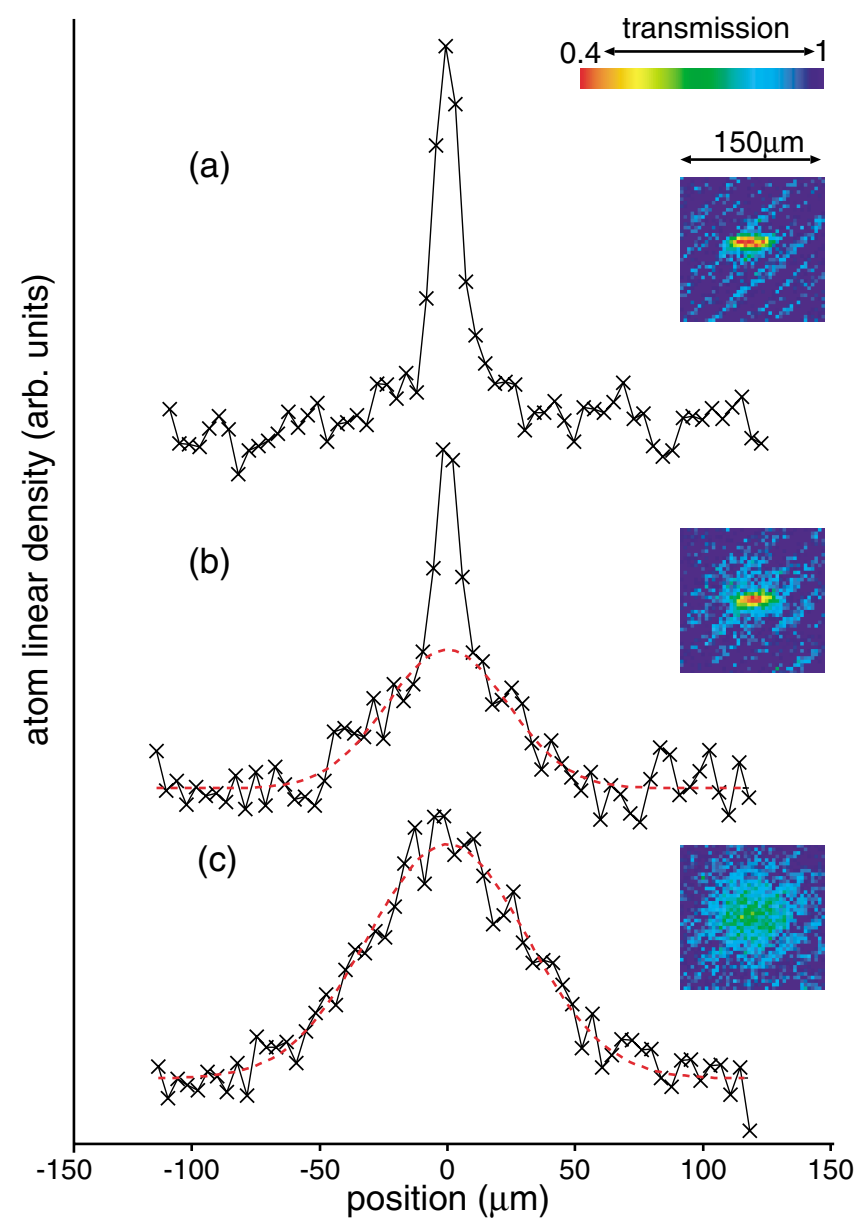

FIG. 3 (color). Density profiles of three sample of ${ }^{174} \mathrm{Yb}$ along the vertical direction after $5 \mathrm{~ms}$ of free expansion, showing transition to BEC. The corresponding pseudocolor absorption images are also shown. The profiles are obtained by integrating the column atom density distribution along the horizontal axis. The red broken lines show the best Gaussian fits to the wings: (a) pure condensate; (b) mixture of condensate and thermal cloud, $T=0.46 \mu \mathrm{K}$; (c) pure thermal cloud, $T=$ $0.79 \mu \mathrm{K}$.
In the configuration of Fig. 2(a), final trap oscillation frequencies in the horizontal and the vertical direction in the center of the trap are estimated to be 1.6 and $0.33 \mathrm{kHz}$, respectively. The mean trap oscillation frequency $\bar{\omega} / 2 \pi$ in the center of the trap is $0.95 \mathrm{kHz}$, and therefore the critical transition temperature given by $T_{c}=$ $\left(\hbar \bar{\omega} / k_{B}\right)(N / 1.202)^{1 / 3}$ is $0.73 \mu \mathrm{K}$, which is consistent with the temperatures observed in Fig. 3. Here $\hbar$ and $k_{B}$ are Planck's constant divided by $2 \pi$ and Boltzmann's constant, respectively. At the temperature $T_{c}$, the peak atom density $n_{0}$ given by $2.6 / \lambda_{\mathrm{db}}^{3}$, where $\lambda_{\mathrm{db}}$ is the de Broglie wavelength, is $7.0 \times 10^{14} \mathrm{~cm}^{-3}$, which is almost the same as the peak density of $4.7 \times 10^{14} \mathrm{~cm}^{-3}$ obtained for the atoms trapped in the crossed FORT at a higher trap depth and a higher temperature of $100 \mu \mathrm{K}$ [19]. We have measured a lifetime of $\sim 500 \mathrm{~ms}$ for trapped BEC atoms and infer an upper bound for the three-body loss coefficient of $4 \times 10^{-30} \mathrm{~cm}^{6} \mathrm{~s}^{-1}$. The distinct feature of insensitivity to external magnetic field is confirmed by our successful formation and detection of free expansion of ${ }^{174} \mathrm{Yb} \mathrm{BEC}$ in the presence of external magnetic field gradient of about $1 \mathrm{G} / \mathrm{cm}$, which would have significantly influenced the behavior of spinor BEC of alkalilike atoms [20].

A scattering length is a crucial parameter governing the nature of BEC. Unfortunately, the scattering length of ${ }^{174} \mathrm{Yb}$ has not been known even for the sign, thus far, except for our quite recent photoassociation work [21], in which we have inferred the scattering length of ${ }^{174} \mathrm{Yb}$ to be less than $3 \mathrm{~nm}$. Here we carefully estimate the scattering length from time evolution of the expansion of the condensate [22]. Because the resolution of our absorption imaging system is not enough to discuss the vertical expansion, we have adopted the horizontal expansion for the estimation of the scattering length of ${ }^{174} \mathrm{Yb}$. Figure 4 shows the time evolution of the horizontal widths after release from the trap. From the time evolution of the horizontal expansion widths, the chemical potential $\mu$ is estimated to be $0.55 \mu \mathrm{K}$, with the assumption that the scattering length is positive and the interaction is so strong that the condensate is treated in the Thomas-Fermi limit (see also Fig. 4). The obtained chemical potential satisfies the relation $\hbar \omega_{x, y, z} \ll \mu$, which ensures that our assumption is valid. The scattering length $a$ can be derived from the chemical potential $\mu$ as $a=(2 \mu)^{5 / 2} / 15 N \hbar^{2} \bar{\omega}^{3} \sqrt{m}$, where $m$ is the mass of ${ }^{174} \mathrm{Yb}$. The obtained scattering length is $6_{-5}^{+10} \mathrm{~nm}$ and the uncertainty of the estimation mainly comes from the limited resolution of the images and uncertainty in the number of atoms. Taking into account the result of our photoassociation experiment, we conclude that the scattering length is between 1 and $3 \mathrm{~nm}$.

In conclusion, we have observed a BEC of ${ }^{174} \mathrm{Yb}$ atoms by evaporative cooling of the atoms in a crossed optical trap. When the atom number of the condensate is $5 \times 10^{3}$, the transition temperature is $0.73 \mu \mathrm{K}$. We have observed 


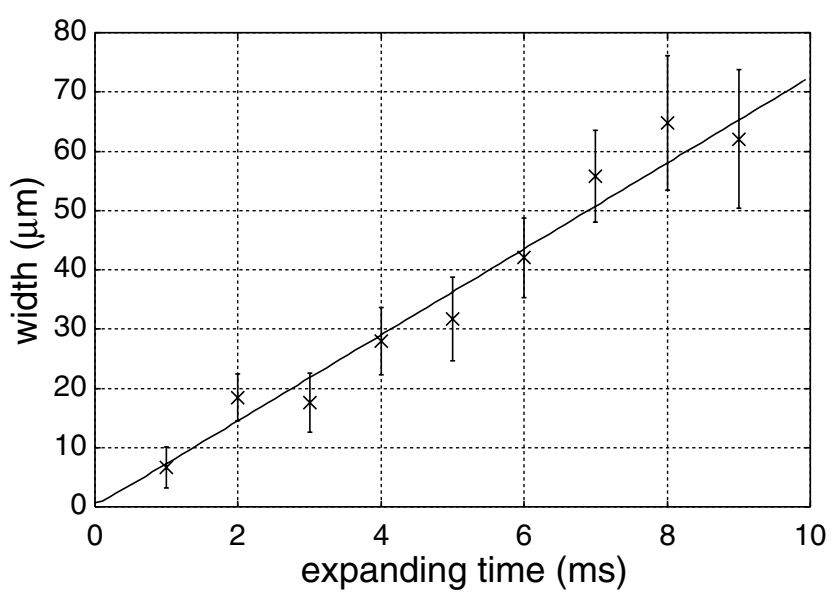

FIG. 4. Measured horizontal widths of condensate whose images are shown in Fig. 2(a), as a function of expanding time. The best fit is also shown as a solid line. The obtained chemical potential is $0.55 \mu \mathrm{K}$.

the bimodal distribution of momentum below the transition temperature. From the time evolution of the horizontal expansion widths, we found out that the condensate is in the Thomas-Fermi limit and the chemical potential is estimated to be $0.55 \mu \mathrm{K}$. The scattering length of ${ }^{174} \mathrm{Yb}$ derived from the chemical potential is $6_{-5}^{+10} \mathrm{~nm}$. Taking into account the result of our photoassociation experiment, we conclude that the scattering length is between 1 and $3 \mathrm{~nm}$.

A natural extension of the present scheme would include formation of BEC of alkaline-earth atoms. Quantum degenerate $\mathrm{Yb}$ atoms would also be quite useful in fundamental physics research such as the test of timereversal symmetry [23,24] and parity violation [25].

This work was partially supported by Grant-in-Aid for Scientific Research of JSPS (11216203, 11640394, 11304023), BT21, REFORT, and JAERI. Two of the authors (Y. Takasu and K. H.) acknowledge support from JSPS.
*Present address: Department of Physics, Tokyo University of Science, Chiba 278-8510, Japan.

${ }^{\dagger}$ To whom correspondence should be addressed. Email address: yitk@scphys.kyoto-u.ac.jp

[1] M. H. Anderson et al., Science 269, 198 (1995).

[2] S. L. Cornish et al., Phys. Rev. Lett. 85, 1795 (2000).

[3] K. B. Davis et al., Phys. Rev. Lett. 75, 3969 (1995).

[4] C. C. Bradley et al., Phys. Rev. Lett. 75, 1687 (1995).

[5] G. Modugno et al., Science 294, 1320 (2001).

[6] T. Weber et al., Science 299, 232 (2003).

[7] D. G. Fried et al., Phys. Rev. Lett. 81, 3811 (1998).

[8] A. Robert et al., Science 292, 461 (2001).

[9] F. P. D. Santos et al., Phys. Rev. Lett. 86, 3459 (2001).

[10] Of course, $\mathrm{He}$ atom has two valence electrons. Metastable $\mathrm{He}\left({ }^{3} \mathrm{He}_{1}\right)$, however, does not have the features such as spinless state and the presence of useful intercombination transition.

[11] J. L. Hall, M. Zhu, and P. Buch, J. Opt. Soc. Am. B 6, 2194 (1989).

[12] C. Henkel and M. Wilkens, Europhys. Lett. 47, 414 (1999).

[13] A. Derevianko et al., Phys. Rev. Lett. 90, 063002 (2003).

[14] A. G. Truscott et al., Science 291, 2570 (2001).

[15] F. Schreck et al., Phys. Rev. A 87, 080403 (2001).

[16] K. Honda et al., Phys. Rev. A 66, 021401 (2002).

[17] M. D. Barrett, J. A. Sauer, and M. S. Chapman, Phys. Rev. Lett. 87, 010404 (2001).

[18] T. Kuwamoto, K. Honda, Y. Takahashi, and T. Yabuzaki, Phys. Rev. A 60, R745 (1999).

[19] Y. Takasu et al., Phys. Rev. Lett. 90, 023003 (2003).

[20] J. Stenger et al., Nature (London) 396, 345 (1998).

[21] Y. Takahashi et al., in Proceedings of the XVIII International Conference on Atomic Physics (ICAP 2002), edited by H. R. Sadeghpour, D. E. Pritchard, and E. J. Heller (World Scientific, Singapore, 2003), p. 83; Y. Takasu et al. (to be published).

[22] Y. Castin and R. Dum, Phys. Rev. Lett. 77, 5315 (1996).

[23] Y. Takahashi et al., in Proceedings of CP Violation and its Origin, edited by K. Hagiwara (KEK, Tukuba, 1997).

[24] A. Dilip et al., J. Phys. B 34, 3089 (2001).

[25] D. DeMille, Phys. Rev. Lett. 74, 4165 (1995). 